\title{
Consideraciones sobre el factor de negociación en las tablas de homologación de avalúos inmobiliarios
}

\author{
Leonardo Joaquín Estrada Ornelas ${ }^{1}$ \\ ${ }^{1}$ Universidad Autónoma de Nuevo León, Facultad de Arquitectura, Maestría en Valuación, Monterrey, Nuevo \\ León, México, leonardo.estrada@ngkf.com, Av. Universidad S/N Col. Ciudad Universitaria, (+52) 554177 \\ 4255
}

Información del artículo revisado por pares

Fecha de aceptación: junio-2021

Fecha de publicación en línea: diciembre-2021

DOI: https://doi.org/10.29105/vtga7.1-126

\section{Resumen}

El factor de negociación en las tablas de homologación de un avalúo comercial de un inmueble es un factor que busca considerar un descuento en función de una negociación comercial, en la medida que el inmueble presenta ciertas características de localización y de uso no habitacional, la experiencia nos invita a considerar un margen mayor en este factor que el de un inmueble habitacional en un mercado en expansión. El análisis realizado a una muestra no probabilística de 31 registros, obtenidos de fuentes primarias de transacciones inmobiliarias en las que el autor ha participado tiene como objetivo compartir esa experiencia mediante un análisis estadístico y en la clasificación de los casos, que permita enriquecer el criterio del factor de negociación para eventos similares. Se encuentra que el factor de negociación en todos los casos es muy superior al $10 \%$ utilizado de forma convencional como un caso extraordinario.

Palabras clave: avalúo inmobiliario, factor de negociación, tabla de homologación.

\section{INTRODUCCIÓN}

La mecánica de valuación inmobiliaria tiene

\begin{abstract}
The negotiation factor of a commercial real estate appraisal discounts the properties' value based on market negotiations. As a property possesses certain characteristics of location and non-residential use, our experience suggests that this negotiation factor is higher in an expanding residential market cycle. The analysis made by 31 non-probabilistic entries, obtained by primary sources of real estate transactions in which the author has participated, has the objective to show that experience, through statistical analysis and classification of cases, will lead to enrich the criteria for further similar events. It has been found that in all cases the negotiation discount factor is considerably higher than $10 \%$ and is used conventionally as an extraordinary case.
\end{abstract}

Keywords: real estate appraisal, negotiation factor, homologation table.

JEL: R20, R33

un proceso definido para estimar el valor de un inmueble, así como se marca en la Norma Oficial Mexicana (NMX) para servicios, 
servicios de valuación y metodología (NMX-R-081-SCFI-2015) existe un mecanismo definido para asegurar que los análisis de la información de mercado y el procedimiento es llevado a cabo de forma ordenada, así como la presentación y los requisitos mínimos que deben incluir. $\mathrm{La}$ sección de análisis de inmuebles comparables se realiza mediante un proceso de homologación a través de unas tablas que evalúan el nivel de similitud, las cuales buscan comparar los aspectos puntuales entre inmuebles que tienen diferencias cuantificables tales como forma, topografía, ubicación y urbanización. Hernández (2017) argumenta que éstas tienen como objetivo equilibrar y sobre todo equiparar las condiciones de los inmuebles considerados como comparables para lograr cierta estandarización y nivelación para utilizarlos de manera objetiva.

Bajo este contexto, la finalidad del presente estudio es identificar los criterios para ponderar con mayor experiencia el factor de negociación principalmente en ciertos tipos y categorías de inmuebles en función del uso, temporalidad del inmueble, ubicación económicamente deprimida o en expansión, son percibidos por el mercado como factores que afectan directamente el precio dispuesto a pagar y el riesgo que se percibe como mayor o menor sea el caso particularmente de cada uno.

Derivado de la forma en la que se obtiene la información de mercado, es decir, mediante una investigación en la que se buscan inmuebles comparables en la zona que compartan similitudes cualitativas y cuantitativas. La hipótesis es que en ocasiones la información de mercado al ser principalmente de precios de salida (asking price) puede sesgar de forma significativa cualquiera estimación. Esto provocaría que la información que se obtenga esté tan alterada o sea tan poco objetiva que, aunque se cumpla con la mecánica y el proceso de valuación, llevará a concluir una estimación muy alejada de la realidad comercial.

El objetivo del presente estudio es que, mediante casos reales podamos enriquecer la consideración del factor de negociación, sobre todos aquellos en los que predomina la experiencia, como es el caso del factor de negociación, en el que, a mayor y mejor información, enriquece el criterio del valuador y sus estimaciones. La muestra no probabilística de este análisis está basada en casos reales y muestra tanto la estimación de valor y el precio de cierre o precio final de compraventa y sus diferencias porcentuales, así como algunas características propias de cada uno de los que buscaremos sintetizar para obtener una clasificación y mediante la aplicación de estadísticos y una regresión lineal aplicada a la diferencia entre valores de salida y de cierre como variable dependiente. La pregunta que se busca responder con la aplicación de estos métodos en la base de datos es si es posible aplicar un criterio más amplio en el factor de negociación bajo diversas circunstancias perfectamente definidas.

Para esto será necesario explicar brevemente los componentes de un avalúo inmobiliario. Salas (2014) señala que se debe comprender los términos básicos y tener presente la diferencia conceptual entre valor y precio, ya que en valuación existe una correlación entre los conceptos de valor y precio, el primero es el resultado de una estimación, de una mecánica y análisis que concluye un posible valor con una justificación, y el precio es el importe que se solicita por un bien o servicio ofertado, el precio de cierre será aquella cifra en la que el mercado está dispuesto a formalizar la transacción de compra venta.

En valuación inmobiliaria la mecánica tradicional solicita que se realice tres consideraciones (NMX-R-081-SCFI2015), una primera producto de una estimación física, la segunda sobre una estimación de mercado y la tercera como una consideración de ingresos. Este estudio se enfoca principalmente en la consideración de mercado, en enriquecer la experiencia para ponderar el factor de negociación, que es producto de la praxis y del intercambio de información entre los actores involucrados en una transacción inmobiliaria. Buscamos ofrecer mejores 
herramientas para poder responder a la pregunta si estamos considerando de la mejor manera los factores de negociación para las tablas de homologación para ser lo más certeros posibles en la estimación final.

\section{MARCO TEÓRICO}

Si bien la historia de la valuación inmobiliaria tiene desde su origen una praxis muy definida en la estimación de valores físicos y que quienes la realizan sobre todo tienen una formación académica muy estrecha con la construcción. La información de mercado es clave para mejorar la estimación bajo la consideración de mercado, es decir, a mayor y mejor información, la estimación puede estar menos sesgada o ser más precisa. Tiene quizá poco más de sesenta años que se considera el entorno de mercado y de capitalización en los avalúos. Hay que recordar que este método de mercado adquiere su información de indicadores de mercado que podemos clasificar como valores de salida, es decir, en valores en los que se anuncia la oferta de un bien inmueble. El tema es que, en los mercados con abundante información, por el tamaño de economía y tipo de producto, esa información puede ser suficiente para realizar estimaciones más o menos certeras (Sánchez, 1986).

El problema surge cuando la información de mercado es limitada por el tipo de producto, el tamaño de mercado o factores que afectan la región, la información disponible no permite tener la certeza de que la estimación tenga un rango de sesgo menor, al contrario, puede estar basada en supuestos completamente alejados del mercado real, del verdadero mercado en el que el precio es el equilibrio entre la oferta y la demanda (Salas, 2015).

Fitch y Soto (2013) en su estudio sobre modelación hedónica en los precios de la vivienda, me dan la pauta para suponer que también en la determinación de los precios de los productos inmobiliarios no habitacionales existen ciertas consideraciones que determinan que el precio sea diferente al estimado y en las próximas líneas tendremos que mostrar como algunos factores determinan que el cruce entre la oferta y la demanda de un inmueble no habitacional esté determinado por diferentes atributos producto de percepciones. También Moreno y Alvarado (2011) hace un estudio sobre precios hedónicos en la vivienda, en el que los atributos incrementan el interés por cierto producto más allá de un mero sentido económico, quizá sería momento de realizar un estudio en inmuebles no habitacionales y si es viable aplicar el criterio de precios hedónicos en estos. Como veremos más adelante, el criterio de selección se basa sobre factores cuantitativos, por lo que no tengo la certeza de que tanto podría utilizarse este análisis en esta categoría de inmuebles.

Por su parte, Prieto (2011) comenta en su estudio sobre las consideraciones de valor histórico y artístico en los trabajos de valuación inmobiliaria, de la necesaria especialización del valuador en ese campo, por todo el contexto y experiencia que esto requiere para una estimación más acertada, así como un método propio para esa categoría de inmuebles, también los demás segmentos del mercado inmobiliario requieren de una mayor especialización. Cada inmueble es diferente y entre segmentos distintos los casos son absolutamente distintos, son mercados con poco en común, son fuentes de información diferentes y por esto mismo la especialización es recomendable para cada uno de los segmentos inmobiliarios, y la importancia de estar generando estudios y documentos que recopilen la experiencia como es el caso o que enriquezcan la práctica y con espíritu crítico se busquen constantemente mejores vías para ofrecer información de mayor calidad.

Antes de entrar en temas de metodología de valuación o de clasificación de eventos, quiero mencionar y recordar el concepto de elasticidad-precio de la demanda de un bien, porque al final los inmuebles son bienes y presentan el fenómeno de la elasticidad, unos más sensibles que otros, o ciertas categorías de 
inmuebles más sensibles a la elasticidad y la intención es entender por qué algunos inmuebles pueden tener una demanda más inelástica, elasticidad generada por la variación en el precio y por la variedad de opciones alternativas.

Si bien este estudio se basa para inmuebles no habitacionales, donde los criterios financieros y objetivos de selección son principalmente cuantitativos, en los que podríamos entender más claro el concepto de elasticidad-precio en cada uno de ellos. La elasticidad explicada por Samuelson y Nordhaus (2002) es la variación de la cantidad demandada al presentarse variaciones en el precio (elasticidad-precio), indicándose porcentualmente cuanto varia la cantidad demandada de un bien al presentarse variaciones en el precio.

Figura 1. Desplazamiento de la oferta y la demanda de mercado en expansión.

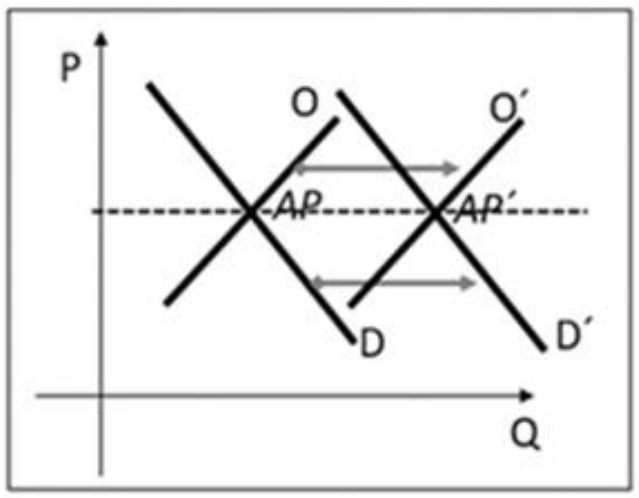

Fuente: elaboración propia.

Siguiendo con las definiciones de Samuelson, se dice que es "elástica" cuando la cantidad demandada responde extraordinariamente a las variaciones en el precio como en los bienes necesarios para vivir y cuando esa respuesta es casi imperceptible en la cantidad demandada ante la variación del precio se dice que es "inelástica" como en los bienes de lujo, agrego, como en los bienes inmuebles no habitacionales. Aunque tradicionalmente se utilizan ejemplos con bienes perecederos o de primera necesidad, no hay que olvidar que los bienes inmuebles, primero son bienes, y algunos de ellos son necesarios, sobre todo los habitacionales y cierto segmento de la vivienda, y presentan cierta inelasticidad de la demanda según el segmento en los que se clasifica la vivienda. En los bienes inmuebles no habitacionales, son sobre todo elásticos, porque hay diversas opciones de localización, de requerimientos técnicos y principalmente, deben ser rentables.

Ha llegado a tal grado la búsqueda de la rentabilidad en los bienes inmuebles, que nuestro desarrollo urbano, está orientado hacia la rentabilidad (Delfante, 2006). Al ser una actividad económica, la industria del desarrollo inmobiliario debe ser rentable. En todo caso, la decisión particular de cada individuo es la que puede buscar parámetros no cuantitativos en los inmuebles, entonces toda aquella decisión que sea principalmente para uso personal (principalmente para vivienda) al estar cargada de esa búsqueda de atributos en el inmueble que se desea sí puede explicarse en gran medida con un análisis de precios hedónicos como los mencionados en los estudios de los párrafos anteriores.

Representar la elasticidad gráficamente puede servir para dar mayor soporte y entender este concepto en nuestro análisis y en concreto en nuestra base de datos. Partimos de los conceptos de oferta y demanda y su representación mediante sus curvas, el primer cuadro es la explicación como el punto de intersección es el precio (Estrada, 2019) y el segundo esquema es el caso donde no hay una intersección por la 
escasa elasticidad, es decir, por ser productos elásticos. Figura 1.

Sin embargo, los casos que analizaremos no son exactamente productos de un mercado en expansión, ya que al ser tan particulares sus elasticidades precio son muy limitadas. Como se muestra en la Figura 2 en la que un producto $\mathrm{Q}$ con una elasticidad de la demanda tan reducida, por diversos factores, que el producto es prácticamente elástico y no es posible tener un precio de cierre, es decir, gráficamente que no se cruzan sus curvas de la demanda y de la oferta en un precio, y por tanto en la realidad tan poco hay un precio de cierre de una transacción.

Figura 2. Elasticidades Limitadas

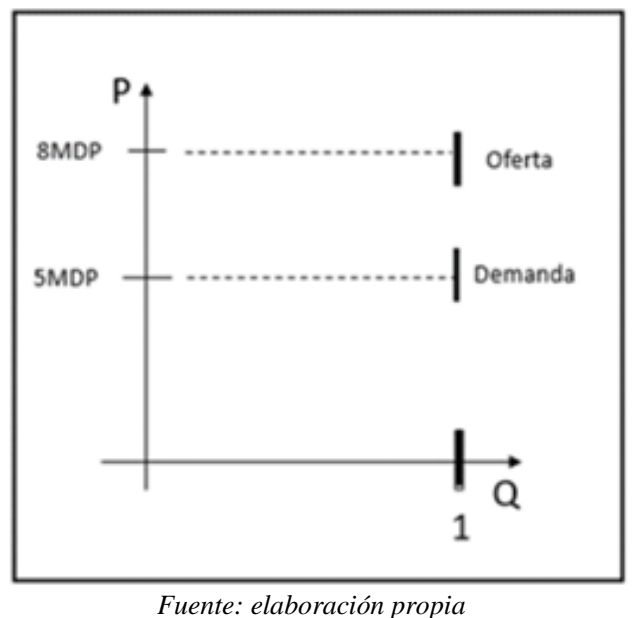

La concepción actual de la valuación inmobiliaria tiene un marco metodológico que considera diferentes vías con sus mecánicas correspondientes para estimar un valor. El método físico, que tiene como objeto calcular el costo neto de reposición de un inmueble en el estado en que se encuentre en el momento del avalúo. El método de mercado que establece el valor de cambio de los inmuebles bajo la premisa de que son necesarios o requeridos por el mercado. El método residual indica que se puede integrar un valor de cambio que integre una unidad en un momento dado, debido al valor del uso o cambio de éste, siempre y cuando sea físicamente posible, legalmente permitido y financieramente viable. El método de capitalización es el enfoque que se basa en la capacidad que tiene un inmueble de generar una renta en el transcurso de un tiempo (Antuñano, 2006).

En la sección de análisis de mercado de los avalúos, que es donde se utiliza la homologación, ya que el factor de homologación es obtener un operador lineal (Hernández, 2017) que relacione el valor de un objeto comparable con un precio conocido con el probable valor del objeto de la valuación. En las tablas en las que se representa esta homologación, se pondera cada factor $y$ al final se obtiene un resultante, en la medida que este criterio sea más objetivo, quizá también el estimado sea más cercano a las consideraciones de mercado.

Las tablas de homologación pueden tener variaciones, pero tienen un común denominador en su elaboración, según la NOM deben contener los diversos factores y se ha preparado una esquematización como se muestra en la Tabla 1.

Tabla 1. Tabla de homologación 


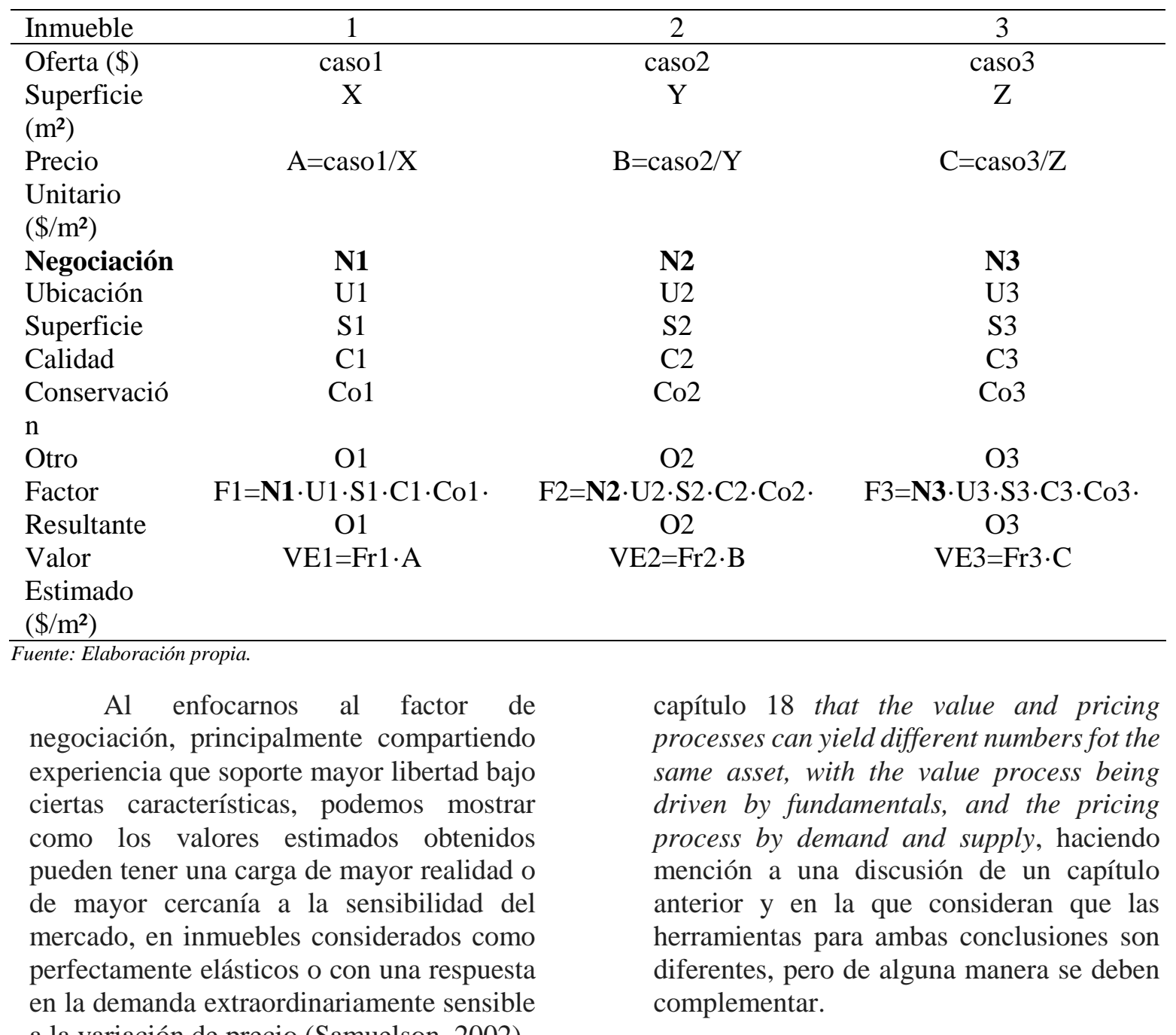

a la variación de precio (Samuelson, 2002).

Damodaran (2018) escribe en el

Figura 3. Análisis de riesgo y repercusión en disminución del precio.

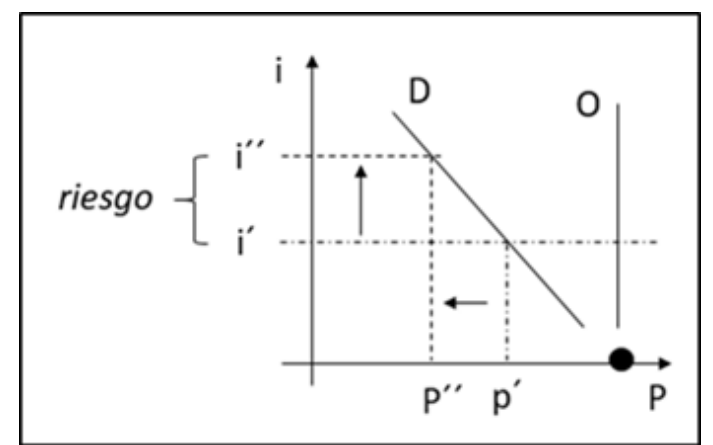

Fuente: elaboración propia.

inmobiliaria está muy definida y tiene su metodología, pero no hay que olvidar que la conclusión es una estimación, y quizá eso es

En efecto, la mecánica de la valuación lo que hay que tener presente. Hay que segmentar el mercado inmobiliario en 
habitacional y comercial, ya que el primero tiene unas consideraciones diferentes, el segmento comercial donde se agrupan todos nuestros casos de estudio están fuertemente vinculados a la rentabilidad y por ende a la oferta y la demanda y por los que hay que tener consideraciones diferentes a las utilizadas en el segmento habitacional.

Cuando hablamos de inmuebles comerciales hay que hablar de parámetros de rentabilidad y por tanto de riesgo, y nos dará un criterio diferente en el que se considere el riesgo. Como mostramos en el esquema adjunto la percepción del incremento del riesgo disminuye el precio. El incremento de i'a i" reducirá de p'a p". Figura 3.

En el caso contrario al anterior, los atributos reales que incrementan la potencialidad o la posibilidad de multiproyectos, por lo que provoca un posible incremento en el precio, pero hasta cierto punto. El descenso en interés de i' por la factibilidad de generar un proyecto de mayor valor agregado puede disminuir a i" y por tanto puede existir un movimiento en Precio de p' a p" pero hasta cierto punto. En ambos casos el precio de venta $\mathrm{P}$, puede estar fuera de cualquier movimiento de la curva por optimista que sea el escenario, en otros casos como el primero, puede ser imposible que jamás se entrecrucen, hasta que se genere un crecimiento de expansión por el tiempo, pero a largo plazo todo puede pasar y también no quiere decir que algún día llegará a ese Precio P. Figura 4.

\section{MÉTODO}

La presente base de datos trata de diferentes inmuebles en los que se tiene la certeza de su valor de avalúo y su valor de cierre, en ellos puede verse la diferencia entre una cifra y otra y podemos determinar algunas características en común que nos permiten inferir que en inmuebles con esas características podríamos tener un criterio más abierto para ponderar el factor de negociación.

Podemos sintetizar las variables y la descripción de éstas en el análisis en la Tabla 2.

Tabla 2. Variables

\begin{tabular}{ll}
\hline \multicolumn{1}{c}{ Variable } & \multicolumn{1}{c}{ Definición } \\
\hline Inmueble & El nombre del inmueble, el identificador del caso. \\
Estimación de Avalúo & Estimación de valor realizada en un momento específico. \\
Fecha de elaboración & Fecha en la que se realiza el avalúo. \\
Precio de cierre & Precio pagado por el inmueble del caso. \\
Fecha de cierre & $\begin{array}{l}\text { Fecha en la que se realiza firma la transacción y el pago por el inmueble } \\
\text { del caso. }\end{array}$ \\
Descuento & Diferencia que se registra entre la estimación de valor y el precio de cierre \\
Tipo & Categoría de Inmueble: I-Industrial, E-Equipamiento, C-Comerciales, D- \\
& Desarrollo, K-Campestre. \\
Economía & Nivel de dinamismo económico en la región, indicadores económicos. \\
& Puede ser A-Alto dinamismo económico, B-Medio dinamismo \\
& económico, C-Bajo dinamismo económico. \\
Localidad & Nivel de comerciabilidad, es decir, grado de dinamismo de la demanda. \\
A-Región muy atractiva para el mercado, B-Región media atractiva para \\
el mercado, C-Región poco atractiva para el mercado.
\end{tabular}

Las variables estimación de avalúo y

precio de cierre son valores expresados 
monetariamente, son entradas de eventos específicos, ya sea por un estudio de valor y de una operación de compra venta respectivamente. Son información objetiva de mercado con una fecha concreta y perfectamente identificable. La variable del descuento es un resultado de las anteriores, representada en una forma porcentual. Ninguna de estas está sujeta a criterio o valoraciones de un individuo. La variable de tipo también es objetiva, es la segmentación adecuada o la categorización de un producto, es la clasificación del inmueble en las diversas actividades o usos para los que fueron diseñados y construidos.

Las variables que denominamos economía y localidad sí están sujetas a una valoración de un perito o analista. Ambas podrían ser consideradas como valoradas en un estudio o ejercicio de valuación, ya que esté contempla la edad del inmueble y su ubicación geográfica, pero la manera en que enfocamos el análisis en estas dos variables va más allá de una dirección y de una vida útil.

La variable economía trata de estimar el nivel de actividad económica de la región, ya que esta califica el dinamismo comercial. Trata de ponderar lo atractivo de invertir en esa región o de operar en esa región, califica la actividad económica desde un punto de vista macroeconómico, ya que esto se vincula al riesgo y como hemos explicado el nivel de percepción de riesgo influye directamente en el precio.

La variable localidad busca ir más allá que una dirección o un polígono, es decir, calificar urbanamente y la actividad económica de una sección geográfica muy específica. Es decir, hay regiones muy atractivas económicamente, pero hay colonias dentro de esas regiones que son muy poco atractivas para invertir, o que quizá están pasando por un período de incertidumbre o son afectadas por proyectos urbanos o entornos socioeconómicos $\mathrm{y}$ viceversa.

La variable novedad es diferente a la edad utilizada en los avalúos, ya que pondera no sólo la vida útil o remanente, sino lo que pondera es la vida del proyecto en su conjunto, es decir, si ha tenido diversos propietarios, diversos usos, transformaciones esenciales, y evidentemente no se trata de un producto inmobiliario nuevo.

La base de datos de elaboración propia (Tabla 4) tiene treinta y un casos en diferentes estados federativos y diferentes características, que sirvió como muestra no probabilística de fuentes primarias, predominan los inmuebles industriales y en todos los casos no son productos nuevos, sino que tienen varios años de uso por el mismo usuario o diferentes propietarios. Ninguno de los casos son inmuebles habitacionales, o aquellos en los que se podría determinar una Demanda constante o producto de un gran mercado de posibles compradores.

La regresión lineal realizada con la base de datos, no resulto estadísticamente significativa y la correlación resultó muy débil, el nivel de significancia no fue significativo. De alguna manera la base de datos está agrupada en un cierto tipo de productos previamente catalogados y las variables califican algo que ya sabemos, por eso la regresión lineal no es significativa, pero los estadísticos descriptivos explicarán el comportamiento de la base de datos.

\section{RESULTADOS}

El resumen de los estadísticos descriptivos (Tabla 3) en todos los casos en los que el producto que además de comercial (no habitacional), es de segunda mano, por improductivo o por reconversión, la posibilidad de que la diferencia sea significativa se incrementa, además de ubicarse en regiones con economías con un bajo atractivo económico que incrementan esas diferencias, ya que el mercado percibe un riesgo más alto, con rendimientos menos atractivos o a plazos más largos que lo hacen ser calificado con un riesgo mayor y que la "palanca" para controlarlo o nivelarlos será el precio, como vemos en los últimos dos esquemas (Gráfico 3 y Gráfico 4). 
Tabla 3. Estadísticos descriptivos

\begin{tabular}{rlr}
\hline Promedios & Promedio de avalúo & $\$ 56,581,324.05$ \\
\cline { 2 - 3 } & Promedio de precio de cierre & $\$ 37,008,420.90$ \\
& Promedio de diferencia & $-28.09 \%$ \\
Rango & Desviación Estándar & $16.795 \%$ \\
& Máxima diferencia & $-66.67 \%$ \\
Tipo (clasificación) & Mínima diferencia & $4.99 \%$ \\
& Industriales & 14 \\
& Comerciales & 7 \\
& Equipamiento & 7 \\
& Campestre & 1 \\
Dispersión Geográfica & Desarrollo & 2 \\
& Centro & 10 \\
& Norte & 17 \\
& Sur & 4 \\
\hline ración propia & &
\end{tabular}

Fuente: elaboración propia

del precio de mercado.

de $\$ 37,000,000$ de pesos y la diferencia entre valor de estimación y precio de cierre fue de $-28.09 \%$ y si eliminamos los extremos obtenemos un promedio de diferencia de $27.90 \%$. Es decir, puede permitirse el factor de negociación en casos similares márgenes superiores al $10 \%$ que en ocasiones se percibe como un límite superior. La desviación estándar es prácticamente del $17 \%$, todos son factores superiores al $10 \%$.

La consideración de nuestras variables pondera la calidad económica o intensidad de la actividad económica de la región, porque estas variables son calificadas por el grado de riesgo que percibe y que impactará directamente en el precio y buscará ajustar el precio para disminuir el precio de ese producto (Gráfico 3), además de que los inmuebles comerciales por definición son aquellos que pueden o generan un ingreso y por tanto se debe medir su rentabilidad para poder comparar con otras inversiones o productos substitutos.

Además, en estos segmentos el principio de sustitución aplica directamente porque en todos los inmuebles comerciales siempre existirán opciones de inversión, alternativas para inversionistas o usuarios finales. Son inmuebles con precios elásticos y por tanto como se muestra en los casos, siempre hay sustitutos que constantemente se comparan entre diferentes opciones y por eso se refleja en la sensibilidad (elasticidad)

\section{CONCLUSIONES}

El avalúo es un estudio con una mecánica definida para estimar un valor y no precisamente es el precio producto de la oferta y la demanda. Los factores que a criterio se ajustan para homologar comparables pueden enriquecerse con la experiencia de la práctica de valuación.

En la muestra analizada los casos tienen en común que no son productos habitacionales, sino de usos comerciales y todos son productos no nuevos, es decir, reciclados, en desuso, en proceso de desinversión o improductivos. En efecto, la regresión lineal no fue significativa, en parte porque todos los casos eran no habitacionales, con una calificación de novedad baja y en los que predominaba niveles de economía baja, no en todos, si en algunos de ellos. También concluimos que quizá es demasiado ambicioso lograr una ecuación en la que calificando las tres variables subjetivas que mencionamos nos estimen un factor de negociación específico. Pero significa qué si se tienen ponderaciones en las variables analizadas, es muy probable que el factor de negociación pueda ser significativamente mayor a los convencionales utilizados en el segmento habitacional.

El avalúo parte de comparables con 
valores de salida, estos valores podrían ajustarse según la muestra para estimar con esta consideración el factor de negociación y quizá lograr valores de conclusión más cercanos a los precios establecidos por la oferta y la demanda en casos con apreciaciones similares en las variables que mencionamos. Para esto la información de mercado será fundamental y es por eso la necesidad de filtrarla mejor o de evaluarla con mayor criterio cada evento, que con la experiencia y especialización se enriquece.

Tabla 4. Base de Datos

\begin{tabular}{|c|c|c|c|c|c|c|c|c|c|c|}
\hline $\begin{array}{c}\text { Cas } \\
\text { o }\end{array}$ & $\begin{array}{l}\text { Avalúo } \\
\text { MDP }\end{array}$ & Fecha & $\begin{array}{l}\text { Cierre } \\
\text { MDP }\end{array}$ & $\begin{array}{c}\text { Fech } \\
\text { a }\end{array}$ & $\begin{array}{c}\text { Descue } \\
\text { nto }\end{array}$ & $\begin{array}{c}\text { Tip } \\
\text { o }\end{array}$ & $\begin{array}{c}\text { Econo } \\
\text { mía }\end{array}$ & $\begin{array}{c}\text { Locali } \\
\text { dad }\end{array}$ & $\begin{array}{l}\text { Nov } \\
\text { edad }\end{array}$ & Estado \\
\hline 1 & 60.259 & 2014 & 46.000 & 2017 & $23.66 \%$ & $\mathrm{I}$ & $\mathrm{C}$ & A & $\mathrm{U}$ & NL \\
\hline 2 & 84.000 & 2015 & 78.166 & 2017 & $6.95 \%$ & I & B & $\mathrm{C}$ & $\bar{U}$ & Tamps \\
\hline 3 & 37.500 & 2016 & 25.000 & 2017 & $33.33 \%$ & I & $\mathrm{C}$ & $\mathrm{C}$ & $\mathrm{U}$ & Dgo \\
\hline 4 & 27.670 & 2016 & 19.990 & 2017 & $27.75 \%$ & I & $\mathrm{C}$ & $\bar{C}$ & $\bar{U}$ & Dgo \\
\hline 5 & 180.000 & 2014 & 60.000 & 2019 & $61.11 \%$ & I & B & $\bar{A}$ & $\mathrm{U}$ & NL \\
\hline 6 & 88.000 & 2016 & 51.000 & 2017 & $42.05 \%$ & I & B & B & $\mathrm{U}$ & NL \\
\hline 7 & 288.000 & 2012 & 172.800 & 2012 & $40.00 \%$ & I & B & A & $\mathrm{U}$ & Méx \\
\hline 8 & 9.950 & 2015 & 6.000 & 2017 & $39.70 \%$ & $\mathrm{C}$ & $\mathrm{B}$ & $\mathrm{C}$ & $\mathrm{U}$ & Ver \\
\hline 9 & 22.750 & 2017 & 14.622 & 2017 & $35.73 \%$ & $\mathrm{D}$ & B & B & $\bar{U}$ & NL \\
\hline 10 & 112.500 & 2016 & 98.315 & 2017 & $12.61 \%$ & $\mathrm{C}$ & B & B & $\mathrm{U}$ & Sin \\
\hline 11 & 60.000 & 2018 & 43.000 & 2019 & $28.33 \%$ & $\mathrm{E}$ & $\mathrm{C}$ & $\mathrm{C}$ & $\mathrm{U}$ & Coah \\
\hline 12 & 21.793 & 2016 & 21.000 & 2017 & $3.64 \%$ & I & $\mathrm{C}$ & B & $\mathrm{U}$ & Ver \\
\hline 13 & 23.500 & 2017 & 18.500 & 2017 & $21.28 \%$ & $E$ & $\mathrm{C}$ & $\mathrm{C}$ & $\overline{\mathrm{U}}$ & Tamps \\
\hline 14 & 60.000 & 2010 & 30.000 & 2010 & $50.00 \%$ & $\mathrm{E}$ & $\mathrm{B}$ & $\mathrm{B}$ & $\mathrm{U}$ & Méx \\
\hline 15 & 36.000 & 2013 & 24.9 & 2015 & $30.83 \%$ & $\mathrm{E}$ & $\mathrm{B}$ & $\mathrm{B}$ & $\mathrm{U}$ & CDMX \\
\hline 16 & 113.000 & 2012 & 100.000 & 2012 & $11.50 \%$ & $E$ & $\mathrm{~A}$ & $\mathrm{~A}$ & $\mathrm{U}$ & CDMX \\
\hline 17 & 60.250 & 2019 & 50.000 & 2020 & $17.01 \%$ & $\mathrm{I}$ & B & B & $\mathrm{U}$ & Jal \\
\hline 18 & 16.350 & 2019 & 15.500 & 2020 & $5.20 \%$ & I & B & $\mathrm{C}$ & $\mathrm{U}$ & CDMX \\
\hline 19 & 8.000 & 2019 & 5.300 & 2020 & $33.75 \%$ & $\mathrm{C}$ & $\mathrm{C}$ & $\mathrm{C}$ & $\mathrm{U}$ & NL \\
\hline 20 & 7.850 & 2019 & 4.800 & 2020 & $38.85 \%$ & $\mathrm{I}$ & $\mathrm{C}$ & $\mathrm{C}$ & $\mathrm{U}$ & Ver \\
\hline 21 & 2.400 & 2019 & 1.400 & 2020 & $41.67 \%$ & $\mathrm{C}$ & $\mathrm{C}$ & B & $\mathrm{U}$ & BCS \\
\hline 22 & .095 & 2019 & .100 & 2020 & $4.99 \%$ & $\mathrm{~K}$ & $\mathrm{C}$ & $\mathrm{C}$ & $\mathrm{U}$ & Tlax \\
\hline 23 & 30.000 & 2016 & 18.000 & 2017 & $40.00 \%$ & $\mathrm{C}$ & $\mathrm{C}$ & $\mathrm{C}$ & $\mathrm{U}$ & Tlax \\
\hline 24 & 93.306 & 2016 & 71.382 & 2016 & $23.50 \%$ & $\mathrm{I}$ & $\mathrm{B}$ & $\mathrm{B}$ & $\mathrm{U}$ & NL \\
\hline 25 & 45.600 & 2014 & 43.000 & 2014 & $5.70 \%$ & $E$ & $\mathrm{~B}$ & $\mathrm{~B}$ & $\mathrm{U}$ & NL \\
\hline 26 & 12.000 & 2010 & 6.684 & 2010 & $44.30 \%$ & $\mathrm{I}$ & $\mathrm{C}$ & $\mathrm{C}$ & $\mathrm{U}$ & Méx \\
\hline 27 & 9.441 & 2011 & 9.100 & 2012 & $3.62 \%$ & $\mathrm{C}$ & $\mathrm{C}$ & $\mathrm{C}$ & $\mathrm{U}$ & Tamps \\
\hline 28 & 10.546 & 2011 & 7.200 & 2013 & $31.73 \%$ & $\mathrm{I}$ & B & B & $\mathrm{U}$ & Jal \\
\hline 29 & 31.160 & 2011 & 19.000 & 2012 & $39.02 \%$ & $\mathrm{C}$ & B & $\mathrm{B}$ & $\mathrm{U}$ & NL \\
\hline 30 & 13.300 & 2013 & 11.000 & 2014 & $17.29 \%$ & $\mathrm{D}$ & $\mathrm{B}$ & $\mathrm{C}$ & $\mathrm{U}$ & CDMX \\
\hline 31 & 188.799 & 2014 & 75.500 & 2015 & $60.01 \%$ & $\mathrm{I}$ & $\mathrm{B}$ & $\mathrm{A}$ & $\mathrm{U}$ & Qro \\
\hline
\end{tabular}

Fuente: Base de Datos propia

El avalúo en su mecánica tradicional será principalmente un estudio de la oferta, y en los casos en los que la oferta y la demanda van muy de la mano o son muy cercanos (habitacional-expansión) la información será de mucha utilidad y vendrá muy apegada al mercado. En los análisis no habitacionales, -como es el casode productos de segunda mano y con apreciaciones pobres de su economía y localidad, la elasticidad de los precios será mayor y una pequeña variación en el precio puede provocar que se salga de mercado y 
viceversa, porque la demanda de esos bienes tiene un comportamiento limitado y volúmenes bajos de operación, por eso el factor de negociación no puede ser como el de un mercado ordinario o en expansión.

La muestra que se obtuvo de los eventos, es producto de la labor profesional del autor y en las que participo en la intermediación de cada uno de los eventos $\mathrm{y}$ es por eso que se conoce a detalle cada uno de ellos y se cuenta con esta información. Podemos decir que es una muestra no probabilística de una base de datos propia.

El propósito era demostrar como bajo algunos criterios adicionales, los factores de negociación en la tabla de homologación puede ser mayor al convencional, que aunque no se logra realizar un modelo estadísticamente significativo, sí permite obtener atributos comunes de ciertos inmuebles en los que sí existe una diferencia significativa entre la estimación del avalúo y el valor de cierre, los cuáles los mencionamos en nuestras variables, que a manera de conclusión son: una región o zona económicamente poco atractiva, un producto no habitacional y una etapa de madurez del producto mismo, atributos que incrementan la sensación de riesgo y que abren la puerta a analizar productos alternativos.

A manera de conclusión podríamos decir que los inmuebles están sujetos a diversos tipos de percepciones que califican de manera negativa, como en la muestra utilizada, su estimación de valor, que van más allá que las variables tradicionales o escapan a ellas y generan una demanda muy baja por ellos y por tanto un desplazamiento a la baja en el precio para encontrar un equilibrio con la oferta.

Como última conclusión, es importante no olvidar que los objetos, empresas o mercados tienen un valor intrínseco, pero al final el que determina el precio final es el mercado mismo en cuanto a una oferta y demanda existente. 


\section{REFERENCIAS}

Antuñano, Antonio. (2006). El avalúo de los bienes raíces. México. Limusa

Bartillac, René. (2015). Ciudades en Bancarrota. CDMX. México. L. D. Books

Damodaran, Aswath. (2018). The dark side of valuation. EUA. Person Education.

Delfante, Charles (2006). Gran Historia de la Ciudad, de Mesopotamia a Estados Unidos. España. Abada Editores.

Estrada-Ornelas, Rosas-Moreira \& Sotelo-Ahumada, (2019). Comportamiento en los precios de arrendamiento del mercado de oficinas en la zona metropolitana de Monterrey. Vinculatégica, 5(2), ISSN 2448-5101.

Fitch, Jesús Manuel, Soto, Karina y Garza, Ricardo. (2012). Valuación de la calidad urbanoambiental. Una modelación hedónica: San Nicolás de los Garza, México, Estudios demográficos y urbanos, 28(“), ISSN 2448-6515.

Hernández, Enrique. (2017). Valuación Inmobiliaria. México, Trillas.

Hull, John. (1999). Options, futures \& other derivatives. EUA. Prentice Hall.

Moreno, R. y Alvarado, E. (2011). El entorno social y su impacto en el precio de la vivienda: un análisis de precios hedónicos en el área metropolitana de Monterrey. Trayectorias, 14(33), 131147.

Norma Oficial Mexicana. Servicios-Servicios de Valuación-Metodología. NMX-R-081-SCFI-2015.

Prieto González, José Manuel (2011). Consideraciones de los valores históricos, artísticos y estéticos en los procesos de valuación inmobiliaria. ACE, 6(16), 31-68. ISSN1886-4805.

Salas, José Manuel, (2015). El modelo de valuación inmobiliaria en México. Revista Iberoamericana para la investigación y desarrollo económico. 5(10) enero-junio 2015.

Salas, José Manuel. (2014). La valuación inmobiliaria tradicional: un modelo para repensar. Paakat. 4(6), marzo-agosto 2014.

Samuelson, Nordhaus. (2002). Economía. España. McGrawHill.

Sánchez, Rafael. (1986). Historia Moderna de la Valuación en la República Mexicana. SHCPINDAABIN. En: https://www.gob.mx/cms/uploads/attachment/file/186123/HISTORIA_MODERNA_SHCP.p df

Varian, Hal. (1998). Microeconomía intermedia un enfoque actual. España. Antonio Bosch editor. 